\title{
Arterial Thrombosis in Cancer: Spotlight on the Neglected Vessels
}

\author{
MARIA LAURA CANALE ${ }^{1}$, IRMA BISCEGLIA ${ }^{2}$, CHIARA LESTUZZI $^{3}$ and IRIS PARRINI ${ }^{4}$ \\ on behalf of ANMCO Cardio-Oncology Task Force \\ ${ }^{1}$ Division of Cardiology, Azienda USL Toscana Nord-Ovest, Ospedale Versilia, Lido di Camaiore, Italy; \\ ${ }^{2}$ Integrated Cardiology Services, Azienda Ospedaliera San Camillo-Forlanini, Rome, Italy; \\ ${ }^{3}$ Cardiology Unit, Oncology Department, CRO, National Cancer Institute, Aviano, Italy; \\ ${ }^{4}$ Division of Cardiology, Ospedale Mauriziano, Turin, Italy
}

\begin{abstract}
Cancer patients are at risk for both venous and arterial thrombotic events. Accumulating evidence suggests a link between cancer and arterial thrombosis events. The pathophysiology of arterial thrombosis in cancer is complex and multifactorial. The risk of arterial thrombosis in cancer patients relies on individual risk factors, on cancer-related hypercoagulability, on anticancer drugs and radiotherapy often via a common underlying mechanism of endothelial dysfunction. This review describes the mechanisms involved in the development of arterial thrombotic events and their clinical manifestations. Furthermore, it provides an overview on therapeutic agents associated with arterial thrombosis.
\end{abstract}

Improvements in anti-cancer global strategy resulted into a better outcome of a large percentage of cancer patients many of which experience definitive cure or long-term survival. As a consequence, toxicities claim attention and physicians should also focus on the long-term management of druginduced side effects mainly related to the cardiovascular system. Cancer is a risk factor for both venous (VTEs) and arterial thrombotic (ATEs) events. The risk of VTEs in cancer patients is clearly defined with a roughly 7 -fold increase compared to the general population (1).

Arterial thrombotic events are increasingly reported in cancer patients both during active treatment and follow-up with various clinical manifestations. The appearance of ATEs

Correspondence to: Maria Laura Canale, Cardiology, Versilia Hospital, Via Aurelia 335 - 55043 Lido di Camaiore (LU), Italy. Tel: +39 05846059721, Fax: +39 05846059719, e-mail: marialaura.canale@uslnordovest.toscana.it

Key Words: Arterial thrombosis, chemotherapy, radiotherapy, angiogenesis, review. is associated with a worse prognosis and a 3-fold increase in overall mortality risk and a probability of recurrent thromboembolism of $37 \%$ at six months $(2,3)$. According to National Cancer Institute's Common Toxicity Criteria, arterial thromboembolism adverse events in cancer patients are related to myocardial ischemia or infarction, cerebral infarction, cerebrovascular accident, cerebral ischemia, ischemic stroke, and peripheral or visceral arterial thrombotic events so highlighting the wide spectrum of possible clinical presentations (4).

Tumor cells directly and indirectly induce a state of hypercoagulability through distinct molecular pathways. Platelet activation, increased synthesis of procoagulant factors and reduction of fibrinolytic activity are all triggers for ATEs. Moreover, local and systemic inflammatory stimuli can turn the endothelium into a pro-thrombotic surface. All these mechanisms could be amplified by anticancer drugs and radiotherapy.

\section{Epidemiology}

Epidemiology of arterial thrombosis has received more attention in recent years. Navi et al., using the Surveillance, Epidemiology, and End Results (SEER) database from 2002 to 2011, have analyzed the incidence of ATEs (as arterial thromboembolic events, myocardial infarction or stroke) in a large population of 279,719 of newly diagnosed cancer patients (2). Compared to an age and sex matched control group they found a cumulative incidence of arterial thromboembolism of $4.7 \%$ in all cancer patients $v s .2 .2 \%$ at 6 months. The risk is higher in some types of tumours such as lung, gastric, and pancreatic. Advance stage was associated with increased ATEs risk, with stage IV patients having a $>10$-fold increase in ATE in the first month after diagnosis of cancer (2). 
A cohort study performed in Taiwan on 52,089 patients with lung cancer and 104,178 matched controls has shown an 1.5 increase in the risk of ischemic stroke in cancer patients (5). This higher risk showed a time trend with a decrease after the first year in men and after the second year in women.

A personal history of cancer-related ATE is associated with a subsequent higher risk of recurrence of thrombosis. Cancer patients with acute ischemic stroke showed a high rate of recurrent ATEs of 21,31 , and $37 \%$ at 1,3 , and 6 months, respectively, with adenocarcinoma having the highest rates of recurrence (3). Grilz et al., in an update of CATS study, have reported an overall incidence of arterial thromboembolism of $2,6 \%$ at two years of observation more frequently reported in lung and kidney cancer patients (6). Interestingly, classic cardiovascular risk factors such as age, male sex, and smoking were found to be independent risk factors for ATE in the CATS cohort.

\section{Pathogenesis}

The pathogenesis of ATEs in cancer is multifactorial and includes individual cardiovascular risk, comorbidities, cancer site and stage and anticancer drugs or radiotherapy.

Cancer cells can express procoagulant factors. Tissue factor (TF) is the receptor for factor (F) VII and site of activation of FVII and FVIIA. TF results in activation of coagulation cascade when it comes into contact with activated FVIIa resulting in local generation of thrombin, the most potent platelet activator (7). An alternative coagulation pathway is via cysteine protein (CP) that activates FX in the absence of FVII. Activation of procoagulant factors can occur during apoptosis of tumor cells induced by chemotherapeutic agents, hypoxia, radiation or haematological growth factors $(7,8)$. Moreover, a reduction in fibrinolysis is involved in tumor growth and metastasis (1). Cancer can activate the coagulation cascade in several ways including the induction of platelets, monocytes and endothelial cells to express a procoagulant phenotype. A reduction in molecules such as antithrombin, proteins $\mathrm{C}$ and $\mathrm{S}$, and tissue plasminogen activator can contribute to the development of arterial thrombosis in cancer $(7,8)$.

Tumor cells can produce ligands including thrombin, ADP, thromboxane A2, metalloproteinases (MMPs) and TF. In response, the stimulation of platelet surface receptors (e.g., PAR-1 and PAR-4 receptors, P2Y12 receptor, and thromboxane receptor) can mediate platelet activation $(8,9)$. Sound evidence indicates increased levels of markers of platelet activation in cancer, including soluble P-selectin, soluble CD40 ligand, platelet factor 4. Cancer leads neutrophils to release decondensed chromatin with formation of extracellular traps so promoting inflammation and thrombosis. Platelets play a pivotal role in tumor progression and metastasis by promoting a hypercoagulability state and, as a consequence, thrombocytosis is a maker of cancer associated thrombosis $(9,10)$.

\section{Clinical Presentation}

Arterial thrombosis may occur in any vessel including limb, myocardial and brain. Clinical presentation of arterial thromboembolism can be acute or subacute, leading to ischemia or infarction with symptoms related to the affected organ. As previously stated, the risk of arterial thromboembolism depends on older age, common cardiovascular risk factors and both cancer and treatmentrelated factors (11). Stroke or myocardial infarction may be the first manifestation of malignant tumours when their cause is not clear (12). The presence of a hypercoagulable state (high levels of D-dimer, diffuse intravascular coagulation) could suggest the need of a screening for undiagnosed cancer. The clinical presentation of ischemic stroke in cancer patients does not grossly differ from that of the general population but sometimes some differences could be identified. A study reported that the percentage of cryptogenic strokes is higher (30 vs. 50\%) in cancer patients with multiple localizations and consequent encephalopathy $(3,13)$. A hypothesis suggests thrombotic endocarditis as possible cause of stroke in cancer patients. This phenomenon could be confirmed by high levels of D-dimer, while it is difficult to be diagnosed in vivo and is more frequently found at autopsy (3). Another possible mechanism for stroke in cancer is paradoxical embolism taking into consideration that about $20 \%$ of cancer patients develop venous thromboembolism or septic embolism (related to infective endocarditis due to the presence of catheters or invasive procedures). Brain tumors remain the main etiology for stroke $(14,15)$.

Cancer patients have a risk of recurrent ischemic stroke approximately 3 -fold higher than non-cancer patients both during active cancer (16) and later due to radiotherapy damage as reported in the Childhood Cancer Survivor Study (17). A recent metanalysis that included 9711 renal cell carcinoma patients from 19 randomized controlled trials (RCT) confirmed an overall incidence of ATE of 1.5\%. The most common events for ATEs were cardiac ischemia/ infarction (67.4\%), CNS ischemia (7.9\%) and cerebrovascular accident $(6.7 \%)$. The highest ATE incidences were noted for cediranib (3.2\%) and pazopanib (2.4\%), and recurrence rates were confirmed to be significantly elevated for pazopanib [4.6 $(95 \% \mathrm{CI}=1.1-18.7)]$ and sorafenib [2.3 $(95 \% \mathrm{CI}=1.2-4.4)]$ only (18).

Peripheral arterial thrombosis is mainly described with the new-generation BCR-ABL inhibitors such as nilitinib, dasatinib and more frequently with ponatinib. Treatmentrelated events may be rapidly progressive and also continue after discontinuation (19). 


\section{Drug-related Arterial Thrombosis}

Angiogenesis inhibitors. Angiogenesis plays an important role in cancer growth, invasion, and metastasis. Inhibition of vascular endothelial growth factor (VEGF) signaling pathway, one of the key paths in cancer neo-angiogenesis control, could lead to endothelial cell damage resulting in vascular toxicity. In clinical practice several angiogenesis inhibitors are utilized for the management of solid and hematologic malignancies.

Anti-VEGF antibodies. Bevacizumab is a recombinant, humanized monoclonal antibody directed against VEGF used in the treatment of many cancer types. ATEs are described with a relatively high frequency but rates vary among trials. In an analysis of pooled data from five RCTs including 1745 patients with metastatic colorectal, breast, or non-small-cell lung carcinoma, the addition of bevacizumab to chemotherapy was associated with an increased risk of ATEs (overall incidence $3.8 \%$ with bevacizumab vs. $1.7 \%$ in the chemotherapy group) (20). In a meta-analysis of 20 RCTs including 12.617 patients with various advanced solid tumors, bevacizumab treatment was associated with an increased risk of all ATEs of 3.3\%. The risk of myocardial infarction was higher with bevacizumab, while no difference was described regarding to ischemic stroke (21). A subsequent meta-analysis of 22 prospective trials enrolling more than 20,000 patients found a higher risk of cardiac and cerebral ischemia in patients receiving bevacizumab compared to chemotherapy. The risk seemed to be directly proportional to the dose of bevacizumab with patients receiving higher doses being at higher risk (22). These findings have not been fully confirmed in a large observational study reporting that the relative risk of ATEs with bevacizumab-based therapy compared with controls was $1.46 \%$ and on subgroup analysis the risk was independent from cancer site, bevacizumab dose or disease stage (23).

It has been proposed that bevacizumab-related endothelial damage with decrease in nitric oxide and PGI-2 synthesis could lead to activation of platelet and coagulation factors resulting in arterial thrombosis (24).

A similar rate of arterial adverse events was reported for aflibercept, a recombinant fusion protein containing VEGFbinding portions fused to the $\mathrm{Fc}$ portion of human immunoglobulin (Ig)G1 used in the treatment of advanced refractory colorectal cancer. All grade and serious arterial thromboembolic adverse events that were reported with a relatively low incidence in VELOUR trial (2.6 and $1.8 \%$ respectively), have been recently confirmed by real word data reporting an incidence of arterial or venous embolic events of $6 \%(25,26)$.

Ramucirumab, a novel fully human IgG1 monoclonal antibody that selectively targets VEGFR-2, was not associated with increased risk of ATE (27).
Immunomodulatory agents. The risk of tromboembolism in patients receiving lenalidomide and pomalidomide especially when administrated with dexametazone or other chemotherapy is high with a global incidence of $8.5 \%$ (28). The mechanism of vascular toxicity is not completely understood. Endothelial damage with reduced production of nitric oxide can led to ischemia/coronary disease and the association with steroids can enhance endothelial stress resulting in platelet activation and thrombosis (28).

Tyrosine kinase inhibitors. Small-molecule inhibitors against receptor tyrosine kinases in cancer cells has dramatically improved survival in several cancer types. In general, the toxicity profile of these drugs differs from those of classic chemotherapy but arterial thrombotic events have been reported. In a meta-analysis by Choueri et al. including 10,255 patients with advanced renal cell carcinoma and other malignancies receiving sorafenib or sunitinib, authors reported an incidence of arterial thrombosis of $1.4 \%$ compared with the control group, with no difference between renal and non-renal cancer or between the two drugs (29). A subsequent meta-analysis of 9,711 patients from 19 RCTs showed an overall incidence of ATEs of $1.5 \%$ (18). The subgroup analyses did not show a significant difference in terms of risk of ATE depending on tumor types, VEGFRtyrosine kinase inhibitors (TKIs), treatment regimens, phase of trials and sample size. Additionally, the most common events for ATEs were cardiac ischemia/infarction (67.4\%), CNS ischemia (7.9\%) and cerebrovascular accident $(6.7 \%)$. Few data are available for others VEGF-TKIs; a comparative meta-analysis of random trials showed no increase in the risk of thrombosis (RR 0.85) for axitinib, cediranib and regorafenib compared to standard treatment (30). A recent systematic meta-analysis focused cardiovascular issues of regorafenib treatment; while hypertension and bleeding were significantly more frequent, arterial thrombosis and heart failure were not (31).

Pazopanib may be responsible for apoptosis of endothelial cells, which promotes coagulation, thus leading to thromboembolic and ischemic events in treated patients. Moreover, its inhibition of VEGF signaling may also increase blood viscosity through overproduction of erythropoietin, exacerbating risk for thrombosis, but ATEs (miocardial infarction/ischemia, cerebrovascular events) are uncommon with pazopanib $(<2 \%)$ in clinical practice (32). To date, no specific data are available about ATEs risk with other VEFG inhibitors as cabozantinib, vandetanib or nintebanib.

$B C R-A B L 1$ kinase inhibitors. Imatinib dramatically changed prognosis of chronic myeloid leukemia (CML) and subsequently gastro-intestinal stromal tumors patients and is considered as a milestone in target therapy development. 
The new-generation BCR-ABL TKIs, designed to overcome imatinib resistance, including nilotinib, dasatinib, bosutinib, and ponatinib are associated with a higher risk of arterial events compared with the standard imatinib (33). Random trials have reported a higher incidence of cardiovascular events with nilotinib treatment compared to imatinib including peripheral arterial occlusive disease and other vascular events (i.e. myocardial infarction and pulmonary embolism). Similar to nilotinib, dasatinib has been associated with a greater rate of ischemic events compared with imatinib. Ponatibib, initially withdrawn by the FDA for high rate of ATEs, can result in peripheral arterial occlusive disease that may be rapidly progressive and these events can continue also after discontinuation of treatment. It may also cause cerebral ischemia and myocardial infarction (19). In patients receiving bosutinib at a lower dose (400 mg), ATEs events can occur less frequently compared with other BCR/ABL1 TKIs, but are still more common than with imatinib. Thrombotic events are more frequent in presence of cardiovascular risk factors but concur also without them. Possible mechanisms of such toxicity have not been clearly established but evidence supports inhibition of ABL kinase with endothelium injury (34).

Antimetabolites. 5-Fluorouracil (5-FU), a pyrimidine analogue, and capecitabine are cornerstones of treatment of gastrointestinal and breast cancers. Ischemic events may occur during infusion or within 2-3 days from the beginning of therapy presenting as angina, acute myocardial infarction, heart failure and cardiogenic shock.

The incidence of ischemia is related to drug dose with higher risk associated to repeated infusions and to administration route. In fact, it is higher during continuous i.v. infusion compared to bolus administration or oral capecitabine. Electrocardiographic changes with S-T depression and $\mathrm{T}$ wave inversion are landmarks of acute ATEs and drug re-challenge is associated with higher risk of cardiac complications (35). The underlying mechanism remains unknown but coronary vasospasm has been reported is several cases; coronary angiography performed immediately after acute event frequently showed no culprit lesions. In that cases, drug infusion could interfere with pathways that mediate vascular smooth muscle tone (36). Another possible mechanism is damage of the arterial endothelium followed by thrombosis (37).

Alkylating agents. Coronary thrombosis is the basis of myocardial infarction associated with platinum compounds. Acute ATEs may develop during or even after cisplatin-based chemotherapy combinations (38). An increased risk of atherosclerotic disease has been described after 10-20 years in testicular cancer survivors, with a percentage ranging from $6 \%$ to $10 \%$ and even higher when treatment was associated with radiotherapy (39). A possible explanation relies on the fact that cisplatin blood levels may remain elevated for many years after therapy discontinuation leading to a long-lasting risk (40). Ischemic stroke is another possible complication of cisplatin-based chemotherapy in young patients probably due to a direct endothelial damage leading to platelet and coagulation cascade activation (41). Together with cisplatin, cyclophosphamide (mainly at high dose) is associated with acute myocardial infarction and stroke. Probably, the two drugs share endothelial damage and platelet adhesion as triggers for toxicity (42).

Radiotherapy. Mediastinum irradiation is associated with several cardiovascular complications. Coronaropathy could be reported long time after exposure, typically 10 to 15 years, mainly involving coronary arteries and distal vessel (43). Cardiovascular toxicity of radiotherapy is dose related. Survivors of Hodgkin lymphoma have 2.7-fold higher risk for coronary heart disease compared to general population (44). Interestingly, when mediastinum is included in treatment field, aorta or great vessels could be targets of late toxicity (45).

Radiotherapy of head and neck tumors could be associated with carotid arteries atherosclerosis with significant internal and common carotid stenosis resulting in transient ischemic attack or ischemic stroke while radiation treatment of brain tumors (mainly involving the circle of Willis) could lead to a later higher risk of stroke in survivors (46).

Independently of the site of treatment, radiotherapy causes early inflammation of the whole arterial wall, followed by vascular fibrosis that can occur in any layer of the vessel wall usually in medium and large size vessels (47). Moreover, irradiation is involved in atherosclerotic plaque formation and accelerates atherosclerosis process (48).

Aromatase inhibitors. Aromatase inhibitors (AIs) are used as adjuvant therapy in hormone receptor-positive postmenopausal early breast cancer patients. A meta-analysis that analyzed switch strategy (tamoxifen followed by AIs) vs. upfront AIs treatment reported a small increase in cardiovascular adverse events with upfront AIs (4.2\% vs. $3.4 \%$ ) (49). Combined analysis of randomized control trials of AIs found a $19 \%$ increase risk of ischemic heart disease compared to tamoxifen. It should be noted that, in the adjuvant setting, tamoxifen was associated with a $33 \%$ decreased risk compared with placebo or no-treatment. Therefore, the increase in cardiovascular risk observed with AIs could be explained, at least in part, by the lack of the protective effect of tamoxifen (50).

\section{Conclusion}

Arterial thrombotic events represent serious and potentially life-threatening adverse events of a variety of anti-cancer 
treatments. Their knowledge and management is of primary importance for both clinical cardiologists and oncologists involved in the care of cancer patients.

\section{Conflicts of Interest}

The Authors have no conflicts of interest to declare regarding this study.

\section{Authors' Contributions}

Conception: MLC, IP; Design: MLC, IP, IB, CL; Literature review and processing: MLC, IP, IB, CL; Writing: MLC, IP, CL; Critical review: MLC, IB, CL; Final approval: MLC, IP, IB, CL.

\section{Acknowledgements}

The Authors are grateful to Miss Lara Camerini for language revision.

\section{References}

1 Gomes $\mathrm{M}$ and Khorana AA: Risk assessment for thrombosis in cancer. Semin Thromb Hemost 40(3): 319-324, 2014. PMID: 24599438. DOI: $10.1055 / \mathrm{s}-0034-1370770$

2 Navi BB, Reiner AS, Kamel H, Iadecola C, Okin PM, Elkind MSV, Panageas KS and DeAngelis LM: Risk of arterial thromboembolism in patients with cancer. J Am Coll Cardiol 70(8): 926-938, 2017. PMID: 28818202. DOI: $10.1016 / \mathrm{j}$.jacc. 2017.06.047

3 Navi BB, Singer S, Merkler AE, Cheng NT, Stone JB, Kamel H, Iadecola C, Elkind MS and DeAngelis LM: Recurrent thromboembolic events after ischemic stroke in patients with cancer. Neurology 83(1): 26-33, 2014. PMID: 24850486. DOI: 10.1212/WNL.0000000000000539

4 National Cancer Institute's Common Toxicity Criteria (version 3). 2006. Available at: https://ctep-cancer-gov.bvs.clas.cineca.it/ protocoldevelopment/electronic applications/docs/ctcaev3.pdf. Last accessed on 11th November 2016.

5 Chen PC, Muo CH, Lee YT, Yu YH and Sung FC: Lung cancer and incidence of stroke: a population-based cohort study. Stroke 42(11): 3034-3039, 2011. PMID: 21903961. DOI: 10.1161/ STROKEAHA.111.615534

6 Grilz E, Königsbrügge O, Posch F, Schmidinger M, Pirker R, Lang IM, Pabinger I, and Ay C: Frequency, risk factors, and impact on mortality of arterial thromboembolism in patients with cancer. Haematologica 103(9): 1549-1556, 2018. PMID: 29794142. DOI: 10.3324 /haematol.2018.192419

7 Blann AD and Dunmore S: Arterial and venous thrombosis in cancer patients. Cardiol Res Pract 3: 394740, 2011. PMID: 21403876. DOI: $10.4061 / 2011 / 394740$

8 Mezouar S, Frère C, Darbousset R, Mege D and Crescence L: Role of platelets in cancer and cancer-associated thrombosis: Experimental and clinical evidences. Thromb Res 139: 65-76, 2016. PMID: 26916298. DOI: 10.1016/j.thromres.2016.01.006

9 Connoly GC, Phipps RP and Francis CW: Platelets and cancerassociated thrombosis. Semin Oncol 41(3): 302-310, 2014. PMID: 25023346. DOI: 10.1053/j.seminoncol.2014.04.009
10 Kedzierska M, Czerner U, Szydlowska-Pazera K, Potemski P, Piekarski J, Jeziorski A and Olas B: The changes of blood platelet activation in breast cancer patients before, after surgery and in various phases of chemotherapy. Platelets 24(6): 462-468, 2013. PMID: 22871094. DOI: 10.3109/09537104.2012.711866

11 Abdel-Qadir H, Ethier JL, Lee DS, Thavendiranathan P and Amir E: Cardiovascular toxicity of angiogenesis inhibitors in treatment of malignancy: A systematic review and meta-analysis. Cancer Treat Rev 53: 120-127, 2017. PMID: 28104567. DOI: 10.1016/j.ctrv.2016.12.002

12 Taccone FS, Jeangette SM and Blecic SA: First-ever stroke as initial presentation of systemic cancer. J Stroke Cerebrovasc Dis 17(4): 169-174, 2008. PMID: 18589335. DOI:10.1016/j.jstroke cerebrovasdis.2008.01.007

13 Schwarzbach CJ, Schaefer A, Ebert A, Held V, Bolognese M, Kablau M, Hennerici MG and Fatar M: Stroke and cancer: the importance of cancer-associated hypercoagulation as a possible stroke etiology. Stroke 43(11): 3029-3034, 2012. PMID: 22996958. DOI: 10.1161/STROKEAHA.112.658625

14 Iguchi Y, Kimura K, Kobayashi K, Ueno Y and Inoue T: Ischemic stroke with malignancy may often be caused by paradoxical embolism. J Neurol Neurosurg Psychiatry 77(12): 1336-1339, 2006. PMID: 16847046.

15 Graus F, Rogers LR and Posner JB: Cerebrovascular complications in patients with cancer. Medicine (Baltimore) 64(1): 16-35, 1985. PMID: 3965856.

16 Kim JM, Jung KH, Park KH, Lee ST, Chu K and Roh JK: Clinical manifestation of cancer related stroke: retrospective case-control study. J Neuroncol 111(3): 295-301, 2013. PMID: 23299460. DOI: $10.1007 / \mathrm{s} 11060-012-1011-4$

17 Fullerton HJ, Stratton K, Mueller S, Leisenring WW, Armstrong GT, Weathers RE, Stovall M, Sklar CA, Goldsby RE, Robison LL and Krull KR: Recurrent stroke in childhood cancer survivors. Neurology 85(12): 1056-1064, 2015. PMID: 26311747. DOI: $10.1212 /$ WNL.0000000000001951

18 Qi WX, Shen Z, Tang LN and Yao Y: Risk of arterial thromboembolic events with vascular endothelial growth factor receptor tyrosine kinase inhibitors: an up-to-date meta-analysis. Crit Rev Oncol Hematol 92(2): 71-82, 2014. PMID: 24878433. DOI: $10.1016 /$ j.critrevonc 2014.04 .004

19 Valent P, Hadzijusufovic E, Schernthaner GH, Wolf D, Rea D and le Coutre P: Vascular safety issues in CML patients treated with BCR/ABL1 kinase inhibitors. Blood 125(6): 901-906, 2015. PMID: 25525119. DOI: 10.1182/blood-2014-09-594432

20 Scappaticci FA, Skillings JR, Holden SN, Gerber HP, Miller K, Kabbinavar F, Bergsland E, Ngai J, Holmgren E, Wang J and Hurwitz H: Arterial thromboembolic events in patients with metastatic carcinoma treated with chemotherapy and bevacizumab. J Natl Cancer Inst 99(16): 1232-1239, 2007. PMID: 17686822.

21 Ranpura V, Hapani S, Chuang J and Wu S: Risk of cardiac ischemia and arterial thromboembolic events with the angiogenesis inhibitor bevacizumab in cancer patients: a metaanalysis of randomized controlled trials. Acta Oncol 49(3): 287297, 2010. PMID: 20156114. DOI: 10.3109/0284186090 3524396

22 Totzeck M, Mincu RI and Rassaf T: Cardiovascular adverse events in patients with cancer treated with bevacizumab: A metaanalysis of more than 20000 patients. J Am Heart Assoc 6(8): pii: e006278, 2017. PMID: 28862931; DOI: 10.1161/JAHA. 117.006278 . 
23 Schutz FA, Je Y, Azzi GR, Nguyen PL and Choueiri TK: Bevacizumab increases the risk of arterial ischemia: a large study in cancer patients with a focus on different subgroup outcomes. Ann Oncol 22(6): 1404-1412, 2011. PMID: 21115602. DOI: $10.1093 /$ annonc/mdq587

24 Yang R, Thomas G, Bunting S, Ko A, Ferrara N, Keyt B, Ross $\mathrm{J}$ and Jin H: Effects of vascular endothelial growth factor on hemodynamics and cardiac performances. J Cardiovasc Pharmacol 27(6): 838-844, 1996. PMID: 8761851.

25 Van Cutsem E, Tabernero J, Lakomy R. Prenen H, Prausová J, Macarulla T, Ruff P, van Hazel GA, Moiseyenko V, Ferry D, McKendrick J, Polikoff J, Tellier A, Castan R and Allegra C: Addition of aflibercept to fluorouracil, leucovorin, and irinotecan improves survival in a phase III randomized trial in patients with metastatic colorectal cancer previously treated with an oxaliplatin-based regimen. J Clin Oncol 30(28): 3499-3506, 2012. PMID: 22949147.

26 Pastorino A, Di Bartolomeo M, Maiello E, Iaffaioli V, Ciuffreda L, Fasola G, Di Costanzo F, Frassineti GL, Marchetti P, Antoniotti C, Leone F, Zaniboni A, Aprile G, Zilocchi C, Sobrero A and Bordonaro R: Aflibercept plus FOLFIRI in the real-life setting: safety and quality of life data from the italian patient cohort of the aflibercept safety and quality-of-life program study. Clin Colorectal Cancer 17(3): e457-470, 2018. PMID: 29605592. DOI: 10.1016/j.clcc.2018.03.002

27 Abdel-Rahman O and El-Halawani H: Risk of cardiovascular adverse events in patients with solid tumors treated with ramucirumab: A meta analysis and summary of other VEGF targeted agents. Crit Rev Oncol Hematol 102: 89-100, 2016. PMID:27129437. DOI: 10.1016/j.critrevonc.2016.04.003

28 Lee DH and Fradley MG: Cardiovascular complications of multiple myeloma treatment: evaluation, management, and prevention. Curr treat Options Cardiovasc Med 20: 19, 2018. PMID: 29508087. DOI: 10.1007/s11936-018-0618-y

29 Choueri TK, Schutz FA, Je Y, Rosenberg JE and Bellmunt J: Risk of arterial thromboembolic events with sunitinib and sorafenib: a systematic review and meta-analysis of clinical trials. J Clin Oncol 28(13): 2280-2285, 2010. PMID: 20351323. DOI:10.1200/JCO.2009.27.2757

30 Abdel-Rahman O and Fouad M: Risk of cardiovascular toxicities in patients with solid tumors treated with sunitinib, axitinib, cediranib or regorafenib: an updated systematic review and comparative meta-analysis. Crit Rev Oncol Hematol 92(3): 194207, 2014. PMID: 25028151. DOI: 10.1016/j.critrevonc. 2014.06 .003

31 Chen J and Wang J: Risk of regorafenib-induced cardiovascular events in patients with solid tumors: A systematic review and meta-analysis. Medicine (Baltimora) 97: e12705, 2018. PMID: 30313066. DOI: 10.1097/MD.0000000000012705

32 Justice CN, Derbala MH, Baich TM, Kempton AN, Guo AS, Ho $\mathrm{TH}$ and Smith SA: The impact of pazopanib on the cardiovascular system. J Cardiovasc Pharmacol Ther 23(5): 387-398, 2018. PMID: 29706106. DOI: 10.1177/107424841 8769612

33 Haguet H, Douxfils J, Mullier F, Chatelain C, Graux C and Dogné JM: Risk of arterial and venous occlusive events in chronic myeloid leukemia patients treated with new generation BCR-ABL tyrosine kinase inhibitors: a systematic review and meta-analysis. Expert Opin Drug Saf 16(1): 5-12, 2017. PMID: 27852118
34 Chopade P and Akard LP: Improving outcomes in chronic myeloid leukemia over time in the era of tyrosine kinase inhibitors. Clin Lymphoma Myeloma Leuk 18(11): 710-723, 2018. PMID: 30093283. DOI: 10.1016/j.clml.2018.06.029

35 Saif MW, Shah MM and Shah AR: Fluoropyrimidineassociated cardiotoxicity: revisited. Expert Opin Drug Saf 8(2): 191-202, 2009. PMID: 19309247. DOI: 10.1517/1474033 0902733961

36 Sudhoff T, Enderle MD, Pahlke M, Petz C and Teschendorf C: 5-fluorouracil induces arterial vasocontractions. Ann Oncol 15(4): 661-664, 2004. PMID: 15033676.

37 Polk A, Vistisen K, Vaage-Nilsen $M$ and Nielsen DL: A systematic review of the pathophysiology of 5-fluorouracilinduced cardiotoxicity. BMC Pharmacol Toxicol 15: 47, 2014. PMID: 25186061. DOI: 10.1186/2050-6511-15-47

38 Doll DC, List AF, Greco FA, Hainsworth JD, Hande KR and Johnson DH: Acute vascular ischemic events after cisplatinbased combination chemotherapy for germ-line tumors of the testyis. Ann Intern Med 105(1): 48-51, 1986. PMID: 2424354.

39 Huddart A, Norman A, Shahidi M, Horwich A, Coward D, Nicholls J and Dearnaley DP: Cardiovascular disease as a longterm complication of treatment for testicular cancer. J Clin Oncol 21(8): 1513-1523, 2003. PMID: 12697875.

40 Gietema JA, Meinardi MT, Messerschmidt J, Gelevert T, Alt F, Uges DR and Sleijfer DT: Circulating plasma platinum more than 10 years after cisplatin treatment for testicular cancer. Lancet 355(9209): 1075-1076, 2000. PMID: 10744098.

41 Periard D, Boulanger CM, Eyer S, Amabile N, Pugin P, Gerschheimer C and Hayoz D: Are circulating endothelialderived and platelet-derived microparticles a pathogenic factor in the cisplatin-induced stroke? Stroke 38(5): 1636-1638, 2007. PMID: 17379819 .

42 Soultati A, Mountzios G, Avgerinou C, Papaxoinis G, Pectasides D, Dimopoulos MA and Papadimitru: Endothelial vascular toxicity from chemotherapeutic agents: preclinical evidence and clinical implications. Cancer Treat Rev 38(5): 473-483, 2012. PMID: 21982720. DOI: 10.1016/j.ctrv.2011.09.002

43 Taylor CW, Povall JM, McGale P, Nisbet A, Dodwell D, Smith JT and Darby SC: Cardiac dose from tangential breast cancer radiotherapy in the year 2006. Int J Radiat Oncol Biol Phys 72(2): 501-507, 2008. PMID:18374500. DOI: 10.1016/j.ijrobp. 2007.12.058

44 van Nimwegen FA, Schaapveld M, Janus CP, Krol AD, Petersen EJ, Raemaekers JM, Kok WE, Aleman BM and van Leeuwen FE: Cardiovascular disease after Hodgkin lymphoma treatment: 40-year disease risk. JAMA Intern Med 175(6): 1007-1017, 2015. PMID: 25915855. DOI: 10.1001/jamainternmed. 2015. 1180

45 De Bruin ML, Dorresteijn LD, van't Veer MB, Krol AD, van der Pal HJ, Kappelle AC, Boogerd W, Aleman BM and van Leeuwen FE: Increased risk of stroke and transient ischemic attack in 5-year survivors of Hodgkin lymphoma. J Natl Cancer Inst 101(13): 928-937，2009. PMID: 19535773. DOI: $10.1093 /$ jnci/djp 147

46 Plummer C, Henderson RD, O'Sullivan JD and Read SJ: Ischemic stroke and transient ischemic attack after head and neck radiotherapy: a review. Stroke 42(9): 2410-2418, 2011. PMID: 21817150. DOI: 10.1161/STROKEAHA.111.615203

47 Brosuius FC 3rd, Waller BF and Roberts WC: Radiation heart disease. Analysis of 16 young (aged 15 to 33 years) necropsy 
patients who received over 3,500 rad to the heart. J Med 70(3): 519-530, 1981. PMID: 6782873.

48 Darby SC, Cutter DJ, Boerma M, Constine LS, Fajardo LF, Kodama K, Mabuchi K, Marks LB, Mettler FA, Pierce LJ, Trott KR, Yeh ET and Shore RE: Radiation-related heart disease: current knowledge and future prospects. Int J Radiat Oncol Biol Phys 76(3): 656-665, 2010. PMID: 20159360. DOI: 10.1016/ j.ijrobp.2009.09.064

49 Amir E, Seruga B, Niraula S, Carlsson L and Ocaña A: Toxicity of adjuvant endocrine therapy in postmenopausal breast cancer patients: a systematic review and meta-analysis. J Natl Cancer Inst 103(17): 1299-1309, 2011. PMID: 21743022. DOI: 10.1093/ jnci/djr242
50 Khosrow-Khavar F, Filion KB, Al-Qurashi S, Torabi N, Bouganim N, Suissa S and Azoulay L: Cardiotoxicity of aromatase inhibitors and tamoxifen in postmenopausal women with breast cancer: a systematic review and meta-analysis of randomized controlled trials. Ann Oncol 28(3): 487-496, 2017. PMID: 27998966. DOI: 10.1093/annonc/mdw673

Received July 26, 2019

Revised August 12, 2019

Accepted August 14, 2019 